\title{
PREVALENCIA DEL SÍNDROME DEL TÚNEL CARPIANO Y FACTORES ASOCIADOS EN ENDODONCISTAS Y ODONTÓLOGOS QUE SE DEDICAN A LA ENDODONCIA Y LABORAN EN BUCARAMANGA Y SU ÁREA METROPOLITANA
}

\author{
${ }^{1}$ Eliana Yesenia Poveda Sánchez, ${ }^{1}$ Martha Cadena Ortiz, ${ }^{1}$ Lina Patricia Rangel Arias, \\ ${ }^{1}$ Maryen Susana Acevedo, ${ }^{2}$ Gloria Cristina Aránzazu Moya,${ }^{3}$ Sergio Ayala Moreno. \\ ${ }^{1}$ Odontóloga U. Santo Tomás, Estudiante de II año de la Especialización en Endodoncia U. Santo Tomás, ${ }^{2}$ Odontologa U. Santo Tomás, \\ Especialista en Patología Oral y Medios Diagnósticos U. Bosque, Docente U. Santo Tomás. ${ }^{3}$ Medico General U. Metropolitana, \\ Especialista en Salud Ocupacional U. Manuela Beltran.
}

Autor responsable de correspondencia: Gloria Cristina Aránzazu M.

Correo electrónico: aranzazugloria@yahoo.es

\begin{abstract}
RESUMEN
Objetivo: Establecer la prevalencia de Síndrome del Túnel Carpiano (STC) en endodoncistas y odontólogos que se dedican a la endodoncia y laboran en Bucaramanga y su área metropolitana.

Materiales y métodos: Estudio observacional analítico de corte transversal. El universo se constituyó por 202 y la muestra por 100 sujetos seleccionados mediante muestreo no probabilístico por conveniencia. La información se obtuvo a través de una encuesta que evaluó los factores de riesgo. El diagnóstico clínico de STC lo realizó un médico laboral por medio de los métodos de Phalen y Tinnel, que fue confirmado a través de electromiografia. Se utilizaron promedios y frecuencias para describir las variables. Se calcularon OR entre las características de interés y STC, y valores de $\mathrm{p}(\mathrm{p}<0.05)$ para establecer significancia estadística.

Resultados: La prevalencia de STC en la totalidad de los odontólogos valorados fue del 12\%; en endodoncistas y odontólogos generales que se dedican a la endodoncia fue de $17.9 \%$ y en odontólogos generales del $8.2 \%$.

Conclusiones: El factor de riesgo estadísticamente significativo para desarrollar STC fue tener una experiencia laboral mayor de 15 años (OR: 6.42, IC 95\%: 1.43-38.94, p = 0.003). [Poveda EY, Cadena M, Rangel MP, Acevedo MS, Aránzazu GC, Ayala S. Prevalencia del síndrome del túnel carpiano y factores asociados en endodoncistas y odontólogos que se dedican a la endodoncia y laboran en Bucaramanga y su área metropolitana. Ustasalud 2009; 8: 101 - 108]
\end{abstract}

Palabras clave: Síndrome del Túnel Carpiano, Odontólogos, Enfermedad laboral, Movimientos repetitivos.

\section{PREVALENCE OF CARPAL TUNNEL SYNDROME AND ASSOCIATED FACTORS IN ENDODONTIC AND GENERAL DENTISTS THAT WORK IN BUCARAMANGA AND ITS METROPOLITAN AREA}

\begin{abstract}
Objective: To establish the prevalence of Carpal Tunnel Syndrome (CTS) in endodontists and dentists who engage in endodontics and work in Bucaramanga and its metropolitan area.

Methods: An observational analytical cross section study was done. The universe was formed by 202 subjects and the sample of 100 selected individuals through non-probabilistic convenience sampling. The information was obtained through a survey that assessed risk factors. The clinical diagnosis of CTS was made by an occupational physician using methods and Tinnel Phalen tests, which was confirmed by electromyography. Averages and frequencies were used to describe the variables. OR were calculated between the characteristics of interest and STC, and $p$ values $(\mathrm{p}<0.05)$ to establish statistical significance.

Results: The prevalence of CTS in all of the dentists rated was $12 \%$, at endodontists and general dentists dedicated to endodontics was $17.9 \%$ and $8.2 \%$ general practitioners.

Conclusions: The statistically significant risk factor for developing STC was having work experience over 15 years (OR: 6.42, 95\% CI 1.43-38.94, $\mathrm{p}=0.003)$.
\end{abstract}

Key words: Carpal Tunnel Syndrome, Dentists, Occupational disease, Repetitive movements.

Recibido para publicación: 12 de septiembre de 2009. Aceptado para publicación: 20 de diciembre de 2009. 


\section{INTRODUCCIÓN}

El síndrome del túnel carpiano (STC) es un estado neuropático a nivel de la muñeca que se asocia con compresión, constricción o irritación mecánica del nervio Mediano de la mano que se usa con mayor frecuencia. ${ }^{1,2}$

La sintomatología del STC se caracteriza por adormecimiento (parestesias), debilidad (pérdida de fuerza muscular), cosquilleo (disestesias), ardor y sensación de edemas (inflamación) en las manos y dedos de predominio nocturno, cuando se encuentra la articulación en reposo, que de no darse manejo oportuno, avanzan a dolores agudos y persistentes, de tal manera que pueden llegar a ser tan incapacitantes que alteran la rutina de trabajo y las actividades diarias. ${ }^{3}$ Genera productividad laboral reducida, pérdida de tiempo en el trabajo, incapacidad temporal o permanente, inhabilidad para realizar las tareas del puesto y un incremento en los costos de compensación al trabajador. ${ }^{4}$ En casos extremos, el STC obliga a las personas a someterse a intervención quirúrgica y a perder muchos días de trabajo o les impide trabajar del todo debido a que el funcionamiento de sus manos se deteriora por completo. ${ }^{3}$

Los factores de riesgo ergonómicos asociados a esta patología incluyen mala postura de la muñeca durante largos periodos, trabajos repetitivos, esfuerzos mecánicos y vibración. ${ }^{5}$ Según el Decreto 1832 de 1994 del Ministerio de Trabajo y Seguridad Social de Colombia, los trabajos con movimientos repetitivos de los dedos, las manos o los antebrazos pueden originar calambre ocupacional de mano o de antebrazo, se considera así al Síndrome del Túnel Carpiano (STC) como enfermedad de origen laboral. ${ }^{6}$

Durante la labor odontológica una de las enfermedades más comunes que afectan la mano y la muñeca de los profesionales es el STC, debido a que en su rutina laboral se realizan actividades manuales con esfuerzos o movimientos repetitivos, apoyos prolongados o mantenidos, posturas forzadas mantenidas de la muñeca, cambios extremos de temperatura y vibración por instrumentos rotatorios. ${ }^{7}$ Por otro lado, influyen múltiples factores, como el número de años de ejercicio, número de pacientes por día, el tipo de trabajo profesional (especialización), la duración de cada jornada, el ciclo de trabajo/descanso. ${ }^{8}$

Los procedimientos que realiza un Especialista en Endodoncia son continuos, marcados y de larga duración por el tiempo que se utiliza para realizar el tratamiento endodóntico (1-3 horas) en cada paciente, lo que genera que los movimientos repetitivos produzcan mayor susceptibilidad al STC. Además, se requieren posturas mantenidas y forzadas con angulaciones marcadas de la muñeca para acceder con mayor precisión al sistema de conductos radiculares del diente. Actualmente con el advenimiento de la tecnología en la endodoncia, durante el procedimiento se manejan instrumentos rotatorios a lo largo de todo el tratamiento lo que genera mayor tensión a los tejidos de la muñeca y por tanto predispone al desarrollo del STC.

En 1997, la Asociación Dental Americana (ADA) reportó que el $9.2 \%$ de los odontólogos habían sido diagnosticados con alguna patología causada por algún tipo de movimiento repetitivo. ${ }^{9} \mathrm{Un}$ estudio realizado por la Universidad de Michigan (1979), en odontólogos determinó que el 33\% de los odontólogos examinados fueron diagnosticados con mononeuropatìa, pero el $32 \%$ de éstos tuvieron síntomas consistentes de STC. Las personas con diabetes, artritis reumatoide y obesidad fueron más susceptibles a mononeuropatìa. ${ }^{10}$

En un estudio realizado en el 2005 , en la ciudad de Bucaramanga y su área metropolitana, se evaluó la prevalencia del síndrome del túnel carpiano y sus factores asociados en odontólogos, y se encontró que la prevalencia del STC fue de 15.8\% y de éstos el $40 \%$ eran endodoncistas. También se observó que el 33\% de los odontólogos que registraron compromiso sistémico y el $25 \%$ de las personas que usaban instrumental manual durante tiempos menores de $240 \mathrm{~min} /$ día presentaban STC, quizás como efecto a las limitaciones que esta condición puede originar. ${ }^{11}$

El objetivo de este trabajo fue establecer la prevalencia de Síndrome del Túnel Carpiano (STC) en endodoncistas y odontólogos que se dedican a la endodoncia, y laboran en Bucaramanga y su área metropolitana.

\section{MATERIALES Y MÉTODOS}

Se realizó un estudio observacional analítico de corte transversal cuya población estuvo conformada por 27 endodoncistas y 175 odontólogos generales que laboraban en Bucaramanga y su área metropolitana durante el año 2009.

El tamaño de la muestra se calculó mediante la rutina Statcal de programa Epi-info versión 6 para estudios de corte transversal, se consideraron las siguientes especificaciones: nivel de confianza del $90 \%$, poder del $80 \%$ prevalencia del STC en odontólogos generales del $16 \%$ y en endodoncistas del $40 \%$ con un RR 0-40 y OR de 0-29 por lo cual una muestra de 100 odontólogos permitiría observar la asociación de interés. La muestra fue seleccionada mediante un muestreo no probabilístico por conveniencia y obtenida a través de la Asociación Santan- 
dereana de Endodoncia, por medio de los referidos personales y del registro obtenido en la base de datos de los directorios telefónicos existentes para la ciudad de Bucaramanga.

Como criterios de inclusión los endodoncistas y odontólogos generales debían tener un año o más de experiencia laboral y que ejercieran su práctica laboral en Bucaramanga y su área metropolitana. Se consideraron como criterios de exclusión los profesionales que presentaran compromiso sistémico por autoreporte, como: enfermedades autoinmunes (artritis reumatoidea, lupus eritematoso, enfermedad de Raynaud), insuficiencia renal, diabetes mellitus, acromegalia, hipotiroidismo, mieloma múltiple, profesionales embarazadas o en periodo de menopausia, obesidad (basado en el Índice de Masa Corporal) y los que sólo se dedicaran a la actividad administrativa.

En esta investigación se aplicó un instrumento tipo encuesta diligenciado por los integrantes del estudio previamente estandarizados donde se registraron las variables edad, género, actividad laboral, tiempo de experiencia laboral como endodoncista, tiempo de experiencia laboral como odontólogo general, cantidad de horas promedio diario que ejerce la endodoncia, cantidad de horas promedio diario que ejerce procedimientos periodontales, cantidad de horas promedio diario que ejerce procedimientos quirúrgicos, movimientos repetitivos realizados por minuto, técnicas de instrumentación y uso inadecuado de guantes.

Previo a la recolección de los datos se efectuó una prueba piloto para estandarizar los procedimientos, definir los tiempos para el desarrollo de las pruebas y el diligenciamiento de la encuesta, para lo cual se contó con la asesoría de un Médico Laboral quien proporcionó una guía sobre el registro de los movimientos repetitivos por minuto realizados por los odontólogos al instrumentar los conductos radiculares y determinar si el uso de los guantes del profesional es el adecuado para el tamaño de su mano. También se realizó la valoración clínica por el médico laboral, quien realizó, a cada uno de los profesionales, las pruebas diagnósticas de Tinnel y Phalen.

La prueba piloto fue realizada en las clínicas odontológicas de la Universidad Santo Tomás en Floridablanca. Esta prueba fue realizada a seis estudiantes de la Especialización en Endodoncia. La encuesta y las pruebas clínicas se ejecutaron en un lapso de tiempo de veinte minutos por cada participante.

Inicialmente, se procedió a la selección, ubicación, coordinación de la aplicación de la encuesta, que fue diligenciada por los integrantes de la investigación y a obtener el respectivo consentimiento informado. Posteriormente y para establecer el diagnóstico de STC, un médico laboral, efectuó las pruebas de Tinnel y Phalen en el consultorio de los participantes del estudio. A los profesionales que presentaron sintomatología para alguna de las pruebas anteriores (Tinnel positivo y Phalen positivo) se les realizó electromiografía para confirmar el diagnóstico de STC que fue realizada en un centro de medios diagnósticos por el fisiatra Juan de Jesús Trillos Vargas (Universidad Javeriana). El equipo utilizado fue de la marca Teca modelo JM.

Para el análisis univariado las variables de razón fueron descritas como promedios y sus correspondientes desviaciones estándar y las variables nominales fueron descritas en porcentajes y su correspondiente Intervalo de confianza del $95 \%$. Para el análisis bivariado se utilizó la prueba $\mathrm{Chi}^{2}$ y también se calcularon las razones relativas de exposición (OR) crudas, mediante regresión logística. También se calcularon valores de $\mathrm{p}$ con un valor de significancia menor de 0.05 para establecer diferencias entre proporciones y significancia de los OR.

Las consideraciones éticas de este trabajo de grado se basaron de acuerdo con la resolución 8430/93 emitida por el Ministerio de Salud en el cual se establecen las normas científicas, técnicas y administrativas para investigación en salud y de la cual se tuvieron en cuenta los siguientes artículos: 7, 8, 14, 15 y 11 literal b. Esta investigación se consideró de riesgo mínimo, ya que se recolectaron datos a través de procedimientos comunes, consistentes en examen físico, una encuesta y una electromiografía.

\section{RESULTADOS}

\section{Análisis univariado}

\section{Características generales}

Se evaluó un total de 100 profesionales de los cuales el 73\% eran de género femenino. El rango de edad de los profesionales osciló entre 25 y 59 años, con un promedio de edad de $39.1 \pm 6.7$ años (Tabla 1).

\section{Características relacionadas con el tiempo de actividad endodóntica}

El promedio de experiencia laboral como endodoncista u odontólogo que se dedica a la endodoncia fue de $11.1 \pm 6.2$ años. El número de horas diarias, en promedio, dedicadas a la endodoncia por los especialistas u odontólogos que se dedican a la endodoncia fue de 4.8 horas, D.E de 2.28 horas (Tabla 1). 
Tabla 1. Características generales de la población de estudio $(n=100)$ y frecuencia de STC, según características de la población de estudio

\begin{tabular}{|c|c|c|c|}
\hline Características & $\begin{array}{l}\text { Global } \\
\text { n (\%) }\end{array}$ & $\begin{array}{c}\text { Presencia de STC } \\
(n=12) \\
n(\%) \\
\end{array}$ & $\mathbf{P}$ \\
\hline \multicolumn{4}{|l|}{ Género $^{\mathrm{a}}$} \\
\hline Femenino & $73(73)$ & $9(75)$ & 0.867 \\
\hline Masculino & $27(27)$ & $3(25)$ & \\
\hline Edad $^{\mathrm{b}}$ (años) & $39.1(6.7)$ & $9(75)$ & 0.038 \\
\hline Mayor 40 años & & $3(25)$ & \\
\hline \multicolumn{4}{|l|}{ Menor o igual de 40 años } \\
\hline \multicolumn{4}{|l|}{ Actividad Laboral $^{\text {a }}$} \\
\hline Endodoncistas y odontólogos dedicados a la endodoncia & 39 (39) & $7(58.3)$ & 0.143 \\
\hline Odontólogos generales & $61(61)$ & $5(41.7)$ & \\
\hline \multicolumn{4}{|l|}{ Tiempo de experiencia como odontólogo ${ }^{b}$} \\
\hline Menor o igual a 15 años & $14.2(6.4)$ & $3(25)$ & 0.003 \\
\hline Mayor de 15 años & & $9(75)$ & \\
\hline $\begin{array}{l}\text { Tiempo de experiencia como endodoncista u odontólogo que se dedica } \\
\text { a la endodoncia }{ }^{\mathrm{b}}(n=39)\end{array}$ & $11.1(6.2)$ & & \\
\hline Número de horas diarias dedicadas a la endodoncia ${ }^{b}(n=39)$ & $4.8(2.2)$ & & \\
\hline Número de horas diarias dedicadas a la cirugía ${ }^{\mathrm{b}}$ & $1.4(0.8)$ & & \\
\hline Número de horas diarias dedicadas a la periodoncia ${ }^{\mathrm{b}}$ & $1.3(0.8)$ & & \\
\hline Técnica de Instrumentación utilizada ${ }^{a}(n=39)$ & & $\mathrm{n}=7$ & \\
\hline Manual & $15(38.5)$ & $5(71.4)$ & 0.047 \\
\hline Combinada & $24(61.5)$ & $2(28.6)$ & \\
\hline \multicolumn{4}{|l|}{ Número de movimientos repetitivos realizados por minuto ${ }^{b}(n=26)$} \\
\hline Menor o igual a 77 movimientos por minuto & $76.7(22.1)$ & $2(16.7)$ & 0.776 \\
\hline Mayor de 77 movimientos por minuto & & $10(83.3)$ & \\
\hline \multicolumn{4}{|l|}{ Uso de guantes ${ }^{a}$} \\
\hline Adecuado & $96(96)$ & & \\
\hline Inadecuado & $4(4)$ & & \\
\hline
\end{tabular}

${ }^{\mathrm{a}}$ : número y porcentaje.

${ }^{\mathrm{b}}$ : promedio y desviación estándar.

\section{Otros factores que influyen en la presencia de} STC en endodoncistas y odontólogos generales que se dedican a la endodoncia

Se determinó que la técnica de instrumentación más utilizada por los endodoncistas y odontólogos generales que se dedican a la endodoncia, evaluados en la muestra, fue la instrumentación combinada (61.5\%). El promedio de movimientos repetitivos realizados por minuto durante el tratamiento endodóntico fue de 76.7 movimientos/minuto con una desviación estándar de 22.1 movimientos (Tabla 1).

\section{Síndrome del Túnel Carpiano a través de dife- rentes métodos de diagnóstico}

En el presente estudio se estableció que el 14\% de los profesionales valorados por el médico laboral presentó sintomatología de Síndrome de Túnel Carpiano
(STC) con el método de Tinnel, el 8\% con el método de Phalen y el $15 \%$ con cualquiera de los dos métodos clínicos. A todos los pacientes que presentaron sintomatología a través de cualquiera de los dos métodos anteriores, el $12 \%$ presentó diagnóstico de STC a través de electromiografia (Tabla 2 ).

De los 14 profesionales que presentaron sintomatología con el método del Tinnel el 50\% eran endodoncistas y odontólogos generales dedicados a la endodoncia, y el 50\% eran odontólogos generales. De los 8 profesionales que presentaron sintomatología con el método del Phalen el 75\% eran endodoncistas y odontólogos generales dedicados a la endodoncia y el $25 \%$ eran odontólogos generales.

\section{Prevalencia de Síndrome del túnel carpiano}

La prevalencia de STC en los participantes de este estudio fué del $12 \%$ (Tabla 2). 


\section{Análisis bivariado}

Al tener en cuenta la actividad laboral, en los 39 endodoncistas y odontólogos generales que ejercen la

Tabla 2. Presencia de Síndrome del Túnel Carpiano (STC) endodoncia, la prevalencia de STC fue del $17.9 \%$ (7) y en los 61 odontólogos generales la prevalencia de STC fué del 8.2\% (5).

\begin{tabular}{lc}
\hline Características & $\mathbf{n}(\%)$ \\
\hline Síndrome del Túnel Carpiano según método de Tinnel & $14(14)$ \\
Síndrome del Túnel Carpiano según método de Phalen & $8(8)$ \\
Síndrome del Túnel Carpiano según cualquiera de los dos métodos clínicos & $15(15)$ \\
Síndrome del Túnel Carpiano según electromiografía & \\
STC Leve & $6(40)$ \\
STC moderado & $6(40)$ \\
STC severo & $0(0)$ \\
Ninguno & $3(20)$ \\
Clasificación del Síndrome del Túnel Carpiano & \\
STC confirmado & $12(12)$ \\
A riesgo de STC & $3(3)$ \\
No STC & $85(85)$ \\
\hline
\end{tabular}

Tabla 3. Frecuencia de STC, según características de la población de estudio ( $\mathrm{n}=12$ )

\begin{tabular}{lc}
\hline Características & $\begin{array}{c}\text { Presencia de STC } \\
\boldsymbol{n}(\%)\end{array}$ \\
\hline Género & $9(75)$ \\
Femenino & $3(25)$ \\
Masculino & $9(75)$ \\
Edad & $3(25)$ \\
Mayor 40 años & \\
Menor o igual de 40 años & $7(58,3)$ \\
Actividad Laboral & $5(41,7)$ \\
Endodoncistas y odontólogos dedicados a la endodoncia & \\
Odontólogos generales & $3(25)$ \\
Tiempo de experiencia como odontólogo & $9(75)$ \\
Menor o igual a 15 años & \\
Mayor de 15 años & \\
Técnica de Instrumentación utilizada (n=7) & $5(71,4)$ \\
Manual & $2(28,6)$ \\
Combinada & \\
Número de movimientos repetitivos realizados por minuto & $2(16,7)$ \\
Menor o igual a 77 movimientos por minuto & $10(83,3)$ \\
Mayor de 77 movimientos por minuto & \\
\hline
\end{tabular}

\section{Características relacionadas con la presencia de Síndrome del Túnel Carpiano}

En este estudio se determinó, en los odontólogos valorados, que tener una edad menor o igual a 40 años no se puede considerar un posible factor protector ni de riesgo frente a la presencia del STC, debido a que el intervalo de confianza pasa sobre 1 (IC
95\%: 0.04-1.12) lo que parece indicar inconsistencia en este resultado, aunque el valor de p muestre significancia estadística (OR: 0.25, IC 95\%: 0.04-1.12, $\mathrm{p}=0.038$ ).

También se estableció que los factores sexo femenino y actividad laboral como endodoncista u odontólogo general que se dedica a la endodoncia son 
posibles factores de riesgo para STC, aunque en este estudio estos hallazgos no fueran estadísticamente significativos.

Con respecto al tipo de instrumentación durante el tratamiento endodóntico, en los odontólogos valorados, realizar instrumentación manual durante la endodoncia no se puede considerar un posible factor protector ni de riesgo frente a la presencia del STC, debido a que el intervalo de confianza pasa sobre 1 (IC 95\%: 0.04-1.12) lo que parece indicar inconsistencia en este resultado, aunque el valor de p muestre significancia estadística (OR: 0.25, IC 95\%: 0.04-1.12, $\mathrm{p}=0.038$ ).
En la investigación la cantidad de movimientos repetitivos por minuto, realizados durante el procedimiento endodóntico, se presentó como un factor protector, no obstante esta asociación no resultó significativa.

El tiempo de experiencia laboral como odontólogo general resultó ser un factor de riesgo, estadísticamente significativo frente al desarrollo de STC, de esta manera tienen 6.42 veces más riesgo de presentar STC quienes llevan más de 15 años en el ejercicio de la odontología que quienes tienen un menor tiempo de desempeño profesional para la ocurrencia de este evento (OR: 6.42, IC 95\%: 1.43-38.94, $\mathrm{p}=0.003$ ) (Tabla 4).

Tabla 4. Factores asociados al Síndrome del Túnel Carpiano

\begin{tabular}{|c|c|c|c|}
\hline VARIABLE & OR & IC $95 \%$ & p \\
\hline \multicolumn{4}{|l|}{ Edad } \\
\hline \multicolumn{4}{|l|}{$>>$ de 40 años } \\
\hline$>\leq \leq$ de 40 años & 0.25 & $0.04-1.12$ & $0.038^{*}$ \\
\hline \multicolumn{4}{|l|}{ Género } \\
\hline \multicolumn{4}{|l|}{$>$ Masculino } \\
\hline$>$ Femenino & 1.12 & $0.25-6.99$ & 0.867 \\
\hline \multicolumn{4}{|l|}{ Actividad laboral } \\
\hline \multicolumn{4}{|l|}{$>$ Odontólogos generales } \\
\hline $\begin{array}{l}\text { Endodoncistas y odontólogos generales que se dedican a la } \\
\text { endodoncia }\end{array}$ & 2.45 & $0.60-10.55$ & 0.143 \\
\hline \multicolumn{4}{|l|}{ Tiempo de experiencia laboral como odontólogo general } \\
\hline$>\leq$ a 15 años & 6.42 & $1.43-38.94$ & $0.003^{*}$ \\
\hline \multicolumn{4}{|l|}{$>>$ a 15 años } \\
\hline \multicolumn{4}{|l|}{ Movimientos repetitivos realizados por minuto } \\
\hline$>\leq$ a 77 movimientos/minuto & 0.78 & $0.14-8.30$ & 0.776 \\
\hline \multicolumn{4}{|l|}{$>\quad>$ a 77 movimientos/minuto } \\
\hline \multicolumn{4}{|l|}{ Técnica de instrumentación } \\
\hline$>$ Instrumentación combinada & 5.5 & $0.71-64.00$ & $0.047^{*}$ \\
\hline$>$ Instrumentación manual & & & \\
\hline
\end{tabular}

${ }^{*} \mathrm{Chi}^{2}, \alpha=0.05$

\section{DISCUSIÓN}

En el presente estudio realizado en endodoncistas y odontólogos que laboran en Bucaramanga y su área metropolitana se encontró una prevalencia de STC del $12 \%$ obtenida a través de exámenes clínicos y de electromiografia. Esta prevalencia fue superior comparada con la encontrada en el estudio realizado por Atroshi y colaboradores, en en el sur de Suecia, en donde se evaluó una población de 2466 sujetos, y la prevalencia de STC tanto clínica como electrofisiológicamente fue de $2.7 \%{ }^{12}$

En la revisión de literatura sobre prevalencia de STC en odontólogos se encontró que en el estudio reali- zado por Hamann y colaboradores sobre prevalencia de STC y mononeuropatia del Nervio Mediano en odontólogos, en el cual se tomó una muestra de 1079 profesionales, el 32\% presentó sintomatología consistente con STC y solo el $2.9 \%$ fue diagnosticado con STC a través del test de electrodiagnóstico. ${ }^{10}$

En algunos estudios realizados en Colombia, se ha observado que en 53 odontólogos que laboraban en la Facultad de Odontología de la Universidad Cooperativa de Colombia en Envigado, Antioquia se presentó una prevalencia de STC de $14.8 \%$ con exámenes clínicos como Phalen, Tinnel, presión, y torniquete. ${ }^{13}$ En el estudio realizado en odontólogos de Bucaramanga y su área metropolitana, la preva- 
lencia de STC con el método clínico de Phalen fue de $15.8 \%,{ }^{11}$ resultados muy similares a los observados en estudio en donde se encontró que la prevalencia de STC con cualquiera de los métodos clínicos (Phalen y Tinnel) fue del $15 \%$.

En Cali, la prevalencia de signos y síntomas del túnel carpiano al usar los métodos de Phalen y Tinnel en odontólogos adscritos a una Entidad Promotora de Salud fue de $37.5 \%,{ }^{14}$ resultado que no coincide con este estudio en el que se presentó que la prevalencia de STC con cualquiera de los métodos clínicos fue del $15 \%$.

Al tener en cuenta la actividad laboral en este estudio, se encontró que la prevalencia de STC en endodoncistas y odontólogos generales que se dedican a la endodoncia fue del $17.9 \%$ resultado que no coincide con el estudio realizado por Rodríguez y colaboradores cuya prevalencia fué del $40 \%,{ }^{11}$ esto probablemente se debió a que la muestra de endodoncistas fue muy pequeña y no tuvieron en cuenta el método diagnóstico electromiográfico. Tampoco coincidió con el de Martínez y colaboradores en donde la especialidad más frecuente de desarrollar STC fue la administración de salud, $17 \%$, seguida de odontología integral del adulto y odontología general, $15 \%$ cada una, cirugía oral, 13\%; el 40\% restante correspondió a odontopediatría, endodoncia, ortodoncia y periodoncia. ${ }^{13}$

Este estudio mostró que la prevalencia de STC confirmada por electromiografia en los 15 odontólogos generales dedicados a la endodoncia fue de $33.33 \%$ (5), mientras en los 24 endodoncistas fue $8.33 \%$ (2). Este resultado podría haberse explicado si se hubiera indagado otro tipo de variables que permitieran confirmar que la realización de actividades diferentes a la endodoncia, generan una mayor predisposición para la presencia de STC en los odontólogos generales que se dedican a la endodoncia (otras actividades tales como cantidad de horas de uso de computador, práctica de algunos deportes, manejo de aparatos que producen vibración).

Es importante resaltar que el tiempo de experiencia laboral mayor de 15 años resultó ser un factor de riesgo estadísticamente significativo frente al desarrollo de STC, debido a que estos profesionales tienen 6.42 veces más riesgo de presentar STC que quienes tienen un menor tiempo de desempeño laboral (OR: 6.42, IC 95\%: 1.43-38.94, $\mathrm{p}=0.003$ ). Bylund y colaboradores evaluaron mujeres expuestas durante su actividad laboral a instrumentos vibratorios y reportaron que después de 6.8 años de exposición se presentaba la primera sintomatología de parestesia en las manos; esta sintomatología fue reportada por el $94 \%$ de higienistas orales y el
$79 \%$ de odontólogas. ${ }^{15}$ Por otro lado, Lalumandier y McPhee observaron en un grupo de higienistas dentales que los que laboraban más de 10 años tenían 1.9 veces más riesgo de presentar STC que los que habían laborado menos años. ${ }^{16}$ En el estudio realizado por Rodríguez colaboradores se encontró que los odontólogos con un ejercicio profesional mayor de 12 años presentaban mayor riesgo de desarrollar STC, comparado con quienes laboraban menos años, sin que se registraran diferencias estadísticamente significativas (R.R: 1.01 IC95\%: 0.48-2.12, $\mathrm{p}=0.962$ ). ${ }^{11}$

$\mathrm{Al}$ tener en cuenta el sexo en este estudio se encontró que las mujeres presentaron mayor riesgo de STC, aunque esta asociación no fue estadísticamente significativa (OR: 1.12, IC 95\%: 0.25-6.99, $\mathrm{p}=0.867$ ) hecho que también se hace evidente en el estudio realizado por Atroshi y colaboradores en donde se observó que una proporción significativa de mujeres presentaron sintomatología al STC $(\mathrm{P}<0.001){ }^{12}$ En el estudio hecho en Montreal por Rossignol y colaboradores se tomó una muestra de 238 pacientes los cuales fueron operados por STC entre junio de 1994 y julio de 1995. En ese estudio se observó que las mujeres presentaron mayor susceptibilidad a la enfermedad debido a que el $27 \%$ fueron hombres y el $74 \%$ fueron mujeres. La proporción hombre mujer fue de 1:3 y la más alta proporción fue encontrada en mujeres sobre la edad de 45 años. ${ }^{17}$ Esto probablemente puede deberse a que el tamaño de la muñeca es más pequeño en mujeres y por tal razón el volumen del canal carpiano es potencialmente más pequeño $o$ a la realización de actividades extralaborales que realizan las mujeres y que pueden predisponerlas para SCT (labores domésticas, otras). ${ }^{10}$ Sin embargo, es de anotar que en este estudio, el número de mujeres participantes fue mayor que el de hombres, hecho que pudo influir en los resultados.

Adicionalmente, se observó que los endodoncistas y odontólogos generales que ejercen la endodoncia son más susceptibles de presentar STC cuando usan instrumentación manual que combinada (manual y rotatoria) pero este factor de riesgo no fue estadísticamente significativo (OR: 5.5, IC 95\%: 0.71-64.00, $\mathrm{p}=0.047$ ). En el estudio de Atroshi y colaboradores, la prevalencia de STC en trabajadores que realizan movimientos repetitivos de la mano o muñeca fue del $2.4 \%$ para aquellos que trabajan más de una hora diaria (IC 95\%: 2.0\%-1.5\%; $p=0.69$ ) y la prevalencia de STC en trabajadores que usan herramientas vibratorias fue del 5.5\% para aquellos que trabajan el mismo tiempo (IC 95\%: 0.0\%-9.1\%; $\mathrm{p}=0.05$ ). ${ }^{11}$

Entre las fortalezas de este estudio se encuentran los métodos de diagnóstico de STC empleados, debido a que se utilizaron las pruebas clínicas de Phalen y 
Tinnel valoradas por un Médico Laboral, Especialista en Salud Ocupacional, cuyo diagnóstico fue confirmado a través de Electromiografía la cual se considera "prueba de oro" para el diagnóstico de STC. Además la encuesta realizada a los participantes del estudio no fue autodiligenciada.

Una debilidad de este estudio fue el tamaño de la muestra en cuanto al número de especialistas en endodoncia y odontólogos generales dedicados a la endodoncia, dado que existen pocos en la ciudad y su área metropolitana, por esta razón se debió incluir a los odontólogos generales.

Además, aunque algunos profesionales estaban interesados en participar en el estudio, ciertos criterios de exclusión como obesidad, embarazo y compromiso sistémico impidieron que pudieran ser parte de esta investigación. Debido al bajo tamaño de muestra, no se pudo establecer la asociación entre la mayoría de factores y la presencia de STC, los cuales posiblemente con un tamaño de muestra mayor hubieran podido resultar factores de riesgo.

Otra gran limitación del estudio, fue la medición de los movimientos repetitivos durante el tratamiento endodóntico, debido a que varios endodoncistas no aceptaron la medición de esta variable, además se observó que durante el tratamiento endodóntico el especialista gasta menos de un minuto cuando realiza movimientos repetitivos sin descanso durante la instrumentación endodóntica.

Entre los factores que pueden aumentar el riesgo de STC, están:

Actividades que requieren movimientos repetitivos de los dedos por largos períodos de tiempo durante el día.

Insuficientes períodos de descanso durante la jornada laboral que sirvan para ejercitar las articulaciones del cuello, hombro y extremidades superiores.

Postura inadecuada al trabajar y al realizar la técnica de trabajo.

Estilo de vida sedentario.

Uñas excesivamente largas.

Sobrepeso y algunas condiciones médicas (diabetes mellitus, artritis, enfermedades tiroideas).

Consumo de alcohol y tabaco.

Ambiente estresante durante la jornada laboral. ${ }^{18}$

\section{BIBLIOGRAFÍA}

1. McCollister C. Surgery of the Musculoskeletal System; Churchill Livingstone, New York, Edinburgh, and London and Melbourne; 1983.
2. Romero E, Ruiz E, Salazar M. Resultados funcionales de pacientes operados por síndrome de túnel del carpo por el servicio de ortopedia y traumatología HALF, segmento de mano durante el periodo de enero del 2005 a noviembre del 2006. [Tesis Posgrado]. Universidad Autónoma de Nicaragua; Nicaragua: 2005-2006. URL disponibile en: http:/www.minsa.gob.ni/bns/monografias/2007/ortopedia/tunel.pdf

3. Parmet $S$, Lynm C, Glass RM. Síndrome del túnel carpiano. JAMA [en línea] 2002; 288. URL disponible en: http:// jama.ama-assn.org/cgi/data/288/10/1310/DC1/1

4. León $\mathrm{N}$, López A. Lesiones músculo esqueléticas en el personal odontológico. Acta Odontol Venez 2006; 44: 413 -418 .

5. Werner RA, Armstrong TJ. Carpal tunnel syndrome: ergonomic risk factors and intracarpal canal pressure. Phys Med Rehabil Clin N Am 1997; 8: 555 - 569.

6. República de Colombia. Decreto 1832 de 1994. Diario Oficial No. 41.473, del 4 de agosto de 1994. Ministerio de Trabajo y Seguridad Social.

7. Valachi B, Valachi K. Preventing musculoskeletal disorders in clinical dentistry: strategies to address the mechanisms leading to musculoskeletal disorders. J Am Dent Assoc 2003; 134: 1604 - 1612.

8. Akkersson I, Lundborg G, Horstmann U, Skerfning S. Neuropathy in female dental personnel exposed to high frequency vibrations. Occup Environ Med 1995; 52: 116 - 123.

9. Ligh RQ. Preventing cumulative trauma injury carpal tunnel syndrome. J Calif Dent Assoc 2002; 30: 671, 674 - 678.

10. Hamann C, Werner RA, Franzblau A, Rodgers PA, Siew C, Gruninger S. Prevalence of carpal tunnel syndrome and median mononeuropathy among dentists. J Am Dent Assoc 2001 ; 132: $163-170$.

11. Rodríguez A, Morales K, Maldonado LP, Jiménez G, Quiroz LF, Concha SC, Rueda SJ. Prevalencia del síndrome del túnel carpiano y sus factores asociados en odontólogos de la ciudad de Bucaramanga y su área metropolitana. Ustasalud 2005; 4: 70 - 73.

12. Atroshi I, Gummesson C, Johnsson R, Ornstein E, Ranstam J, Rosen I. Prevalence of carpal tunnel syndrome in a general population. JAMA 1999; 282: 153 - 158.

13. Martínez E, Arias O, Cuesta DP, Echavarria L, Pareja LM, Mora E. Prevalencia del Síndrome del Túnel Carpiano en odontólogos. Facultad de Odontología Universidad Cooperativa de Colombia, Envigado. Revista de la Facultad de Odontología 2005; 1: 7 - 11.

14. Alonso S, Aponte P, Delgado A, Montoya N, Daza C. Prevalencia de signos y síntomas del túnel carpiano en odontólogos adscritos a una Entidad Promotora de Salud de Cali, año 2006. Journal Odontológico Colegial 2008; 1: 44-47.

15. Bylund SH, Burstrom L, Knutson A. A descriptive study of woman injured by hand-arm vibration. Ann Occup Hyg 2002; 46: 299 - 307.

16. Lalumandier JA, McPhee SD. Prevalence and risk factors of hand problems and carpal tunnel syndrome among dental hygienists. J Dent Hyg 2001; 75: 130 - 134.

17. Rossignol M, Stock S, Patry L, Armstrong B. Carpal tunnel syndrome: what is attributable to work? The Montreal Study. Occup Environ Med 1997; 54: 519 - 523.

18. Ingram-Rice B. Carpal tunnel syndrome: more than a wrist problem. Journal of Bodywork and Movement Therapies 1997; 1: $155-162$. 